\title{
LESSON 115
}

MARGINS: Pica, 20-70; Elite 30-80.

\section{Clinic}

'en' words

If you have difficulty typing words featuring the letters 'en', try this exercise.

\section{Revise numbers}

Test your Skill

7 minutes.

MARGINS: Pica, 10-72; Elite, 15-85.

Keep the right margin as straight as possible.

Use double-line spacing.

Mark all your errors and look up the suggested corrective exercises in the Clinic Index.

Do you always move the carriage to one side when you erase mistakes?

Is your machine one of those that needs cleaning each time the mechanic calls?

$\mathrm{T} 25-\mathrm{SI} 1.31$
Type each line 3 times paying attention to good typing technique.

1. end ten tend bend spend trend fend wend entry mend 2. lend send open penny suspend intend friend vendors 3. men went tent bent envy scent event slender cement

4. fr4 fr4 f4f f4f f44f ju7 ju7 j7j j7j j77j ju7 $f 4 j 7$ 5. Add 4 and 7 and 7 and 4 and 4 and 7 to get the 33 .

6. ft5 ft5 f5f f5f f55f jy6 jy6 j6j j6j j66j jy6 f5j6 7. The 56 boys and 65 girls had 656 sweets from Tony.

8. de 3 de 3 d3d d3d d33d ki8 ki8 k8k k8k k88k k8k d3k8

9. In 8 days the 38 men and 8 women walked 383 miles.

10. sw2 sw2 s2s s2s s22f f2s lo9 lo9 191 199j j9l s 219 11. The 21 cars and 19 lorries went for 991,221 miles.

The method of transport it is. decided to make use of at any given time will depend upon a number of factors. These factors will include: the value of the article being moved, the distance the goods are to be moved and the speed with which they must be delivered. The question of safety has to a big extent been resolved since all modern methods have been rendered safe. There are accidents but these are few in number no matter what system is used to transport goods.

Big heavy, low value goods go by rail if they can since rail transport is best suited for moving these kinds of goods - particularly if they have a long way to go. Rail transport suffers because it is rigid and you can only go where there are rails and stations opened for use. The other problem to be faced with rail transport is the question which arises out of the need to load and unload goods as they move from a lorry to a rail carriage and back to a lorry to be delivered. This can cost time as well as money.

Road transport is very flexible as you can go all over the country by road and it is easy if you want a door-to-door service. Road transport is at its best for journeys under 100 miles and can take all kinds of goods. The canals in this country do not enter into the question except on a very local basis.

\begin{tabular}{|l|l|l|l|l|l|l|l|l|l|l|l|} 
& 1 & 2 & 3 & 4 & 5 & 6 & 7 & 8 & 9 & 10
\end{tabular}

\section{LESSON 115}

